\title{
THE COMBINATORIAL CATEGORY OF ANDERSEN, JANTZEN AND SOERGEL AND FILTERED MOMENT GRAPH SHEAVES
}

\author{
PETER FIEBIG AND MARTINA LANINI
}

\begin{abstract}
We give an overview on the series of articles [FL1, FL2, FL3] that aims at introducing a new approach towards the "combinatorial" category introduced by Andersen, Jantzen and Soergel in their work on Lusztig's conjecture on the irreducible highest weight characters of modular algebraic groups.
\end{abstract}

\section{INTRODUCTION}

One of the essential steps in the proof of Lusztig's conjecture for large enough characteristics is the work of Andersen, Jantzen and Soergel [AJS94]. There, the authors define a category $\mathcal{M}$ over an arbitrary field $k$ that has the property that it encodes important structural information on the representation theory of quantum groups at a root of unity if $k$ is of characteristic zero, and of modular Lie algebras if $k$ is of positive characteristic. Using an intricate base change argument, the authors were able to show that Lusztig's formula for the irreducible highest weight characters of simple, simply connected modular groups follows from the analogous formula in the quantum case (which was proven earlier by Kazhdan and Lusztig), provided that the characteristic of the base field is large enough.

After this seminal result was obtained, representation theorists were hoping that every prime above the Coxeter number of the group might be large enough in the above sense, and many people were aspiring to prove this. However, in 2013 Geordie Williamson came up with a series of counterexamples, and in addition to showing that the above hope was too optimistic, Williamson also showed that the exceptional characteristics grow exponentially with the Coxeter number [Wil]. This result is both discouraging and challenging. So far we seem not to have a good idea of what is going on for the exceptional primes, and we seem to be quite far from stating a new conjecture on the irreducible characters in case the characteristic of the ground field is exceptional.

Lusztig's conjecture was inspired by explicit examples for the irreducible characters of algebraic groups that were found by Jantzen, who was using a variety

Both authors were partially supported by the DFG priority program 1388 "Representation Theory". 
of powerful tools (such as Jantzen's sum formula, see also [Jan77]) for the calculations. In the present situation, we would like to provide further methods and tools. We hope that they will be of use for gaining an understanding of what to expect of modular representation theory at small primes. Now, the AndersenJantzen-Soergel result referred to above holds for any field of characteristic above the Coxeter number, and it allows us to deduce the irreducible highest weight characters from multiplicities encoded in the category $\mathcal{M}$. This motivates our attempt to try to look at the category $\mathcal{M}$ from a new perspective.

There is a precedent for our approach. In the case of the conjecture of Kazhdan and Lusztig on the irreducible highest weight characters of semisimple complex Lie algebras, one can translate the problem into the realm of either the category of Soergel bimodules, or of Braden-MacPherson sheaves on finite moment graphs. The connection to Soergel bimodules is established via translation functors, whereas the connection to sheaves on moment graphs can be obtained more directly, using the fact that Braden-MacPherson sheaves are projective objects inside a certain category of sheaves that admit a Verma flag (cf [Fie08]). Both approaches have their advantages.

In the positive characteristic case, the translation combinatorics side is incorporated in the definition of the Andersen-Jantzen-Soergel category. Now we want to construct the sheaves-on-moment-graphs side of the picture. It turns out that the right category to look at is the category of filtered sheaves on $f$ nite moment graphs. We define this category together with an exact structure and we relate the projective objects to the Andersen-Jantzen-Soergel category. The projective objects can be constructed in two essentially different ways: On the one hand side, there is a filtered Braden-MacPherson algorithm that yields the indecomposable projectives directly. On the other hand, our category also carries translation functors, and the indecomposable projectives occur as direct summands of Bott-Samelson-type objects.

We can relate our category to the category of sheaves on affine moment graphs, and hence are able to obtain multiplicity formulas that imply Lusztig's conjecture for large enough primes.

\section{The Andersen-JantZen-Soergel CATEgory}

The category of Andersen, Jantzen and Soergel that appears prominently in their work on the representation theory of modular Lie algebras and quantum groups, is sometimes called a "combinatorial category". This term might be somewhat misleading, as the category has not much to do with classical, settheoretic combinatorics. It is rather a category that is defined in terms of basic linear algebra and that is meant to "categorify" the algorithm for calculating the periodic polynomials inside the periodic Hecke module. Let us now introduce the basic notions. 
2.1. Alcoves and reflections. Let $R \subset V$ be an irreducible root system in the Euclidean vector space $V$. We denote by $R^{\vee} \subset V^{*}$ the system of coroots, and $\alpha^{\vee} \in R^{\vee}$ is the coroot associated with $\alpha \in R$. With $X \subset V$ we denote the weight lattice. It is acted upon by the (finite) Weyl group $\mathcal{W}$.

We define, for $\alpha \in R$, the affine translation $t_{\alpha}: V \rightarrow V, \lambda \mapsto \lambda+\alpha$. From this we obtain an action of the root lattice $\mathbb{Z} R$ on $V$. The affine Weyl group is defined as the subgroup generated by $\mathcal{W}$ and $\mathbb{Z} R$ inside the group of affine transformations of $V$. The affine Weyl group is also generated by the following subset of affine reflections: For $\alpha \in R$ and $n \in \mathbb{Z}$ we denote by $s_{\alpha, n}: V \rightarrow V$ the map $\lambda \mapsto \lambda-\left(\left\langle\lambda, \alpha^{\vee}\right\rangle-n\right) \alpha$, i.e. the affine reflection at the hyperplane

$$
H_{\alpha, n}:=\left\{\lambda \in V \mid\left\langle\lambda, \alpha^{\vee}\right\rangle=n\right\} .
$$

The set of alcoves $\mathscr{A}$ is the set of connected components of the topological space $V \backslash \bigcup_{\alpha, n} H_{\alpha, n}$ (we think of $V$ as being endowed with its standard, metric topology). Then $\mathscr{A}$ is acted upon by the affine Weyl group $\widehat{\mathcal{W}}$, and this action is free and transitive. Let us fix a system of positive roots $R^{+} \subset R$. We denote by $A_{e} \in \mathscr{A}$ the base alcove, i.e. the unique alcove contained in the dominant Weyl chamber in $V$ and that contains 0 in its closure. The map $\widehat{\mathcal{W}} \rightarrow \mathscr{A}, w \mapsto w\left(A_{e}\right)$, is a bijection, and we define

$$
A_{w}:=w\left(A_{e}\right)
$$

We denote by $\Pi \subset R^{+}$the set of simple roots, and by $\gamma \in R^{+}$the highest root (i.e. the unique element with the property $\gamma-\alpha \in \mathbb{Z}_{\geq 0} R^{+}$for all $\alpha \in R^{+}$). The set of simple affine reflections is

$$
\widehat{\mathcal{S}}:=\left\{s_{\alpha, 0} \mid \alpha \in \Pi\right\} \cup\left\{s_{\gamma, 1}\right\} .
$$

The reflection hyperplanes corresponding to $s \in \widehat{\mathcal{S}}$ are precisely the hyperplanes that have a codimension 1 intersection with the closure of $A_{e}$.

For any $\alpha \in R^{+}$we define a bijection $\alpha \uparrow \cdot: \mathscr{A} \rightarrow \mathscr{A}$. Let $A$ be an alcove. Set $n=n_{A, \alpha}:=\min \left\{m \in \mathbb{Z} \mid\left\langle\lambda, \alpha^{\vee}\right\rangle<m\right.$ for all $\left.\lambda \in A\right\}$. Then we set

$$
\alpha \uparrow \lambda:=s_{\alpha, n}(A) .
$$

We denote by $\preceq$ the minimal partial order on the set $\mathscr{A}$ that satisfies $A \preceq \alpha \uparrow A$ for all positive roots $\alpha$ and all alcoves $A$.

Let $A \in \mathscr{A}$ be an alcove, and let $s \in \widehat{\mathcal{S}}$. Let us denote by $H_{s}$ the reflection hyperplane corresponding to $s$. Then there is a unique reflection hyperplane $H=H_{A, s}$ in the $\widehat{\mathcal{W}}$-orbit of $H_{s}$ that has a codimension 1 intersection with the closure of $A$. Let us denote by $A s$ the image of $A$ under the reflection at $H$. We then have $A s \preceq A$ or $A \preceq A s$, and we denote by $A_{-}^{(s)}$ (by $A_{+}^{(s)}$, resp.) the smaller (larger) alcove in the set $\{A, A s\}$. 
2.2. Localizations. Now let us fix a field $k$. We denote by $S=S\left(X \otimes_{\mathbb{Z}} k\right)$ the symmetric algebra of the $k$-vector space spanned by the lattice $X$. Let us define for any positive root $\alpha$ the localization

$$
S^{\alpha}=S\left[\beta^{-1} \mid \beta \in R^{+}, \beta \neq \alpha\right]
$$

of $S$ and

$$
S^{\emptyset}=S\left[\beta^{-1} \mid \beta \in R^{+}\right] .
$$

We then have canonical inclusions $S \subset S^{\alpha} \subset S^{\emptyset}$ for each positive root $\alpha$.

2.3. The surrounding category. We now have all ingredients to define the "combinatorial" category that surrounds the Andersen-Jantzen-Soergel category.

Definition 2.1 ([AJS94]). Let $\mathcal{K}$ be the category that consists of objects

$$
M=\left(\{M(A)\}_{A \in \mathscr{A}},\{M(A, \beta)\}_{A \in \mathscr{A}, \beta \in R^{+}}\right),
$$

where

(1) $M(A)$ is an $S^{\emptyset}$-module for each $A \in \mathscr{A}$ and

(2) for $A \in \mathscr{A}$ and $\beta \in R^{+}, M(A, \beta)$ is an $S^{\beta}$-submodule of $M(A) \oplus M(\beta \uparrow A)$. A morphism $f: M \rightarrow N$ in $\mathcal{K}$ is given by a collection $\left(f_{A}\right)_{A \in \mathscr{A}}$ of homomorphisms $f_{A}: M(A) \rightarrow N(A)$ of $S^{\emptyset}$-modules, such that for all $A \in \mathscr{A}$ and $\beta \in R^{+}, f_{A} \oplus f_{\beta \uparrow A}$ maps $M(A, \beta)$ into $N(A, \beta)$.

It is convenient to also introduce the following shift functors that incorporate the $\mathbb{Z} R$-symmetry of the set of alcoves. For an element $\gamma \in \mathbb{Z} R$ and an object $M$ of $\mathcal{K}$ we define the functor $\tau_{\gamma}: \mathcal{K} \rightarrow \mathcal{K}$ as follows. For an alcove $A$ and a positive root $\beta$ we set

$$
\begin{aligned}
\left(\tau_{\gamma} M\right)(A) & =M(A+\gamma), \\
\left(\tau_{\gamma} M\right)(A, \beta) & =M(A+\gamma, \beta) .
\end{aligned}
$$

If $f: M \rightarrow N$ is a morphism in $\mathcal{K}$, then $\tau_{\gamma} f: \tau_{\gamma} M \rightarrow \tau_{\gamma} N$ is given by $\left(\tau_{\gamma} f\right)_{A}=$ $f_{A+\gamma}$.

2.4. Translation functors and the base object. In order to define the AndersenJantzen-Soergel category we need a set of translation functors that act on the category $\mathcal{K}$. Let $s \in \widehat{\mathcal{S}}$ be a simple affine reflection and let $M$ be an object in $\mathcal{K}$. We now define an object $T_{s} M$ in $\mathcal{K}$. Let $A$ be an alcove and $\beta \in R^{+}$. We set

$$
\left(T_{s} M\right)(A):=M\left(A_{-}^{(s)}\right) \oplus M\left(A_{+}^{(s)}\right)
$$

and

$$
\left(T_{s} M\right)(A, \beta):= \begin{cases}\{(\beta x+y, y) \mid x, y \in M(A, \beta)\}, & \text { if } \beta \uparrow A_{-}^{(s)}=A_{+}^{(s)} \\ \beta M(\beta \downarrow A, \beta) \oplus M(\beta \uparrow A, \beta), & \text { and } \beta \uparrow A_{-}^{(s)}, \\ & \text { if } A_{-}^{(s)}=A_{+}^{(s)}, \\ M\left(A_{-}^{(s)}\right) \oplus M\left(A_{+}^{(s)}\right), & \text { and } A=A_{+}^{(s)},\end{cases}
$$


These definitions are functorial in $M$ in the obvious way, and hence yield a functor $T_{s}: \mathcal{K} \rightarrow \mathcal{K}$.

Apart from the translation functors we also need the following base object $Q_{0}$ in $\mathcal{K}$. For an alcove $A$ and a positive root $\beta$ we set

$$
Q_{0}(A):= \begin{cases}S^{\emptyset}, & \text { if } A \in \mathcal{W}\left(A_{e}\right), \\ 0, & \text { if } A \notin \mathcal{W}\left(A_{e}\right)\end{cases}
$$

and

$$
Q_{0}(A, \beta):= \begin{cases}\left\{(\beta x+y, y) \mid x, y \in S^{\beta}\right\}, & \text { if } A, \beta \uparrow A \in \mathcal{W}\left(A_{e}\right), \\ \beta S^{\beta}, & \text { if } A \in \mathcal{W}\left(A_{e}\right), \beta \uparrow A \notin \mathcal{W}\left(A_{e}\right), \\ S^{\beta}, & \text { if } A \notin \mathcal{W}\left(A_{e}\right), \beta \uparrow A \in \mathcal{W}\left(A_{e}\right), \\ 0, & \text { if } A, \beta \uparrow A \notin \mathcal{W}\left(A_{e}\right) .\end{cases}
$$

2.5. The Andersen-Jantzen-Soergel category of "special objects" in $\mathcal{K}$. We are now ready to define the category $\mathcal{M}$.

Definition 2.2. The category of special objects is the smallest full subcategory $\mathcal{M}$ of $\mathcal{K}$ that satisfies the properties.

- It contains the object $Q_{0}$.

- It is stable under the translation functors $T_{s}$ for any simple affine reflection $s$ and the shift functors $\tau_{\gamma}$ for any $\gamma \in \mathbb{Z} R$.

- It is stable under taking direct summands and forming direct sums.

2.6. The connection to modular representation theory. Now suppose that $k$ is an algebraically closed field of positive characteristic $p$, and suppose that $G$ is an almost simple, connected and simply connected algebraic group defined over $k$. Let $T \subset G$ be a maximal torus, and suppose that the associated root system is $R$. We can then identify $X$ with the set of weights $\operatorname{Hom}\left(T, k^{\times}\right)$of $G$.

Let $\mathfrak{g}$ be the Lie algebra of $G$, and $\mathfrak{h} \subset \mathfrak{g}$ the Lie algebra of $T$. We now consider a certain category $\mathcal{C}$ of $X$-graded restricted representations of $\mathfrak{g}$. First note that $\mathfrak{g}$ is a restricted Lie algebra, so we can consider the category of restricted representations. An object in $\mathcal{C}$ is now a finite dimensional restricted representation $M$ of $\mathfrak{g}$ that carries an additional grading $M=\bigoplus_{\mu \in X} M_{\mu}$ as a vector space such that the following holds: for $H \in \mathfrak{h}$ and $m \in M_{\mu}$ we have $H . m=\bar{\mu}(H) m$, where $\bar{\mu} \in \operatorname{Hom}(\mathfrak{h}, k)$ is the differential of $\mu$. A morphism of $X$-graded representations is a homomorphism of representations of $\mathfrak{g}$ that is diagonal with respect to the gradings.

An important property of $\mathcal{C}$ is the following: If $M$ is a rational representation, then differentiating the $G$-action yields a representation of $\mathfrak{g}$ on $M$. If we in addition remember the weight space decomposition, i.e. the action of $T$, then we obtain an object in $\mathcal{C}$.

The category $\mathcal{C}$ is an abelian category. For any $\lambda \in X$ one defines the baby Verma module $Z(\lambda)$ in $\mathcal{C}$ with highest weight $\lambda$, and its unique simple quotient 
$L(\lambda)$. We now consider the $p$-dilated and $\rho$-shifted action of the affine Weyl group on the lattice $X$, i.e. we consider the semidirect product $\widehat{\mathcal{W}}_{p}=\mathcal{W} \ltimes \mathbb{Z} p R$ with its natural action on $X$ shifted by $\rho$, i.e. $w \cdot \lambda=w(\lambda+\rho)-\rho$. We identify $\widehat{\mathcal{W}}_{p}$ with the affine Weyl group $\widehat{\mathcal{W}}$ acting on the set $\mathscr{A}$ in the obvious way. Let $w_{0}$ be the longest element in $\mathcal{W}$.

Theorem 2.3 ([AJS94]). Suppose that $p>h$.

- For any alcove $A \in \mathscr{A}$ there is an up to isomorphism unique indecomposable object $Q_{A}$ in $\mathcal{M}$ with $Q_{A}(B)=0$ unless $A \preceq B$, and $Q_{A}(A) \cong S^{\emptyset}$.

- We have $[Z(w .0): L(x .0)]=\operatorname{rk}_{S^{\emptyset}} Q_{A_{w_{0} x}}\left(A_{w_{0} w}\right)$ for all $w, x \in \widehat{\mathcal{W}}$.

(Note that from the construction it follows that for any object $M$ of $\mathcal{M}$ and any alcove $A$, the $S^{\emptyset}$-module $M(A)$ is free of finite rank).

This fundamental result shows that the category of special objects encodes the Jordan-Hölder multiplicities of the baby Verma modules in the category $\mathcal{C}$. In fact, the result obtained in [AJS94] is much stronger: The category $\mathcal{M}$ is even equivalent to the category of (deformed) projective objects in $\mathcal{C}$, hence it encodes the (full) categorical structure of $\mathcal{C}$ !

2.7. Lusztig's conjecture. The affine Weyl group together with the set of simple affine reflections $\widehat{\mathcal{S}}$ is a Coxeter system, so it comes equipped with a length function $\ell: \widehat{\mathcal{W}} \rightarrow \mathbb{Z}_{\geq 0}$ and a Bruhat order $\leq$. The affine Hecke algebra $\widehat{\mathcal{H}}$ is the free $\mathbb{Z}\left[v^{ \pm 1}\right]$-module with basis $\left\{H_{w} \mid w \in \widehat{W}\right\}$ whose algebra structure is uniquely determined by the relations

$$
\begin{aligned}
H_{w} \cdot H_{x} & =H_{w x} \text { if } \ell(w x)=\ell(w)+\ell(x), \\
H_{s}^{2} & =H_{e}+\left(v^{-1}-v\right) H_{s} \text { for } s \in \widehat{\mathcal{W}} .
\end{aligned}
$$

Then $H_{e}$ is a multiplicative identity in $\widehat{\mathcal{H}}$, and it turns out that each $H_{w}$ is invertible in $\widehat{\mathcal{H}}$. The Kazhdan-Lusztig involution on $\widehat{\mathcal{H}}$ is the $\mathbb{Z}$-linear involution - that is determined by $\bar{v}=v^{-1}$ and $\bar{H}_{w}=H_{w^{-1}}^{-1}$. The element $\underline{H}_{s}=H_{s}+v$ is self-dual with respect to this involution for any simple affine reflection $s \in \widehat{\mathcal{S}}$.

The periodic module $\mathcal{P}$ is the free $\mathbb{Z}\left[v^{ \pm 1}\right]$-module with basis $\{A \mid A \in \mathscr{A}\}$, equipped with a right action of $\widehat{\mathcal{H}}$, which is uniquely determined by

$$
A \cdot \underline{H}_{s}= \begin{cases}A s+v A, & \text { if } A=A_{-}^{(s)}, \\ A s+v^{-1} A, & \text { if } A=A_{+}^{(s)},\end{cases}
$$

for all $A \in \mathscr{A}$ and $s \in \widehat{\mathcal{S}}$. Let $\mathcal{P}^{\circ}$ be the $\widehat{\mathcal{H}}$-submodule of $\mathcal{P}$ generated by the set

$$
\left\{E_{\lambda}:=\sum_{w \in \mathcal{W}} v^{\ell(w)}\left(w\left(A_{e}\right)+p \lambda\right) \mid \lambda \in X\right\} .
$$

The following theorem gives us a distinguished basis for the module $\mathcal{P}^{\circ}$. 
Theorem 2.4 ([Lus80b], [Soe97, Theorem 4.3]). (1) There exists a unique additive involutive map $\div: \mathcal{P}^{\circ} \rightarrow \mathcal{P}^{\circ}$ such that $\overline{E_{\lambda} h}=E_{\lambda} \bar{h}$ for all $\lambda \in X$, $h \in \widehat{\mathcal{H}}$.

(2) For any $A \in \mathscr{A}$ there exists a unique element $\underline{P}_{A}$ such that $\overline{\underline{P}_{A}}=\underline{P}_{A}$ and $\underline{P}_{A}=A+\sum_{B \in \mathscr{A} \backslash\{A\}} p_{A, B} B$ with $p_{A, B} \in v \mathbb{Z}[v]$. The set $\left\{\underline{P}_{A}\right\}_{A \in \mathscr{A}}$ is a basis for $\mathcal{P}^{\circ}$.

The $p_{A, B}$ appearing in the above statement are called the periodic polynomials.

Now Lusztig's conjecture can be reformulated (e.g., [Fie10]) as the following statement about the Jordan-Hölder multiplicities of baby Verma modules: For any $x, w \in \widehat{\mathcal{W}}$ we have $[Z(w .0): L(x .0)]=p_{A_{w_{0} w}, A_{w_{0} x}}(1)$.

2.8. An intrinsic definition of $\mathcal{M}$ ? The above result of Andersen, Jantzen and Soergel places the multiplicity problem into a somewhat elementary context. The categories $\mathcal{M}$ and $\mathcal{K}$ are defined using only linear algebraic structures, they do not refer any more to the representation theory of Lie algebras or reductive groups. Yet the construction of $\mathcal{M}$ is quite complicated, as experience shows that it is quite hard to calculate explicitely with the translation functors defined in Section 2.4. It is hence desirable to have an alternative, maybe more intrinsic definition of $\mathcal{M}$.

An example of such an intrinsic definition in a related context is the following. In the case that the characteristic of $k$ is zero, the analogue (and the inspiration) of Lusztig's conjecture is the slightly older conjecture of Kazhdan and Lusztig on the characters of simple highest weight representations of semisimple complex Lie algebras. In the approach of Soergel one translates this problem into a decomposition problem of Soergel bimodules, or, equivalently, of moment graph sheaves. The Soergel bimodules (or the Braden-MacPherson sheaves) can be characterized as being the projective objects in a surrounding category of objects that "admit a Verma flag". This fact yields a translation-functor-free proof of the analogue of Theorem 2.3 in this context (cf. [Fie08]), and it might help to understand similar situations in which translation functors cannot be defined, as for example the restricted category $\mathcal{O}$ at the critical level for an affine complex Kac-Moody algebra.

In the following section we review the articles [FL1],[FL2] and [FL3], in which an alternative construction of the category $\mathcal{M}$ is given.

\section{3. (Co-)Filtered MOdules OVER STRUCTURE ALGEBRAS}

Let us consider the algebra

$$
\overline{\mathcal{Z}}:=\left\{\left(z_{x}\right) \in \bigoplus_{x \in \mathcal{W}} S \mid z_{x} \equiv z_{s_{\alpha} x} \quad \bmod \alpha^{\vee} \text { for all } x \in \mathcal{W}, \alpha \in R^{+}\right\}
$$

This is the structure algebra (over the field $k$ ) of the finite moment graph $\mathcal{G}$ associated to the root system $R$. It is a $\mathbb{Z}$-graded, commutative, unital $S$-algebra. 
3.1. Cofiltered $\overline{\mathcal{Z}}$-modules. Now we consider the set $\mathscr{A}$ as a topological space with the $\preceq$-order ideals as the open sets. That means that $\mathcal{J} \subset \mathscr{A}$ is open if $A \in \mathcal{J}$ and $B \preceq A$ imply $B \in \mathcal{J}$. An $(\mathscr{A}, \preceq)$-cofiltered $\overline{\mathcal{Z}}$-module, as defined in [FL1], is nothing but a sheaf of $\overline{\mathcal{Z}}$-modules on $\mathscr{A}$. Yet we decided not to use this terminology, as we are also considering $(\mathscr{A}, \preceq)$-cofiltered sheaves on the finite moment graph and we would have to call these objects "sheaves on $\mathscr{A}$ of sheaves on $\mathcal{G}$ ", which is a confusing terminology that we want to avoid. So our definition of an $(\mathscr{A}, \preceq)$-cofiltered object $M$ is as follows. It is given by $\overline{\mathcal{Z}}$-modules $M \preceq A$ for all $A \in \mathscr{A}$ together with restriction homomorphisms $r_{A, B}: M^{\preceq A} \rightarrow M^{\preceq B}$ whenever $B \preceq A$. This data should satisfy $r_{A, A}=\operatorname{id}_{M \preceq A}$ for all $A \in \mathscr{A}$, and $r_{B, C} \circ r_{A, B}=r_{A, C}$ if $C \preceq B \preceq A$. We will also use the more suggestive notation $\left.m\right|_{\preceq B}$ for $r_{A, B}(m)$. A morphism $f: M \rightarrow N$ between $(\mathscr{A}, \preceq)$-cofiltered $\overline{\mathcal{Z}}$-modules is given by a family of homomorphisms $f \preceq A: M \preceq A \rightarrow N \preceq A$ for all $A \in \mathscr{A}$ that is compatible with the restriction homomorphisms in the obvious way.

For each such open subset $\mathcal{J}$ and each $(\mathscr{A}, \preceq)$-cofiltered $\overline{\mathcal{Z}}$-module $M$ we then define

$$
M^{\mathcal{J}}:=\left\{\left(m_{A}\right) \in \prod_{A \in \mathcal{J}} M^{\leqslant A}\left|m_{A}\right|_{\preceq B}=m_{B} \text { for all } A, B \in \mathcal{J} \text { with } B \preceq A\right\} .
$$

This is a $\overline{\mathcal{Z}}$-module again. For open subsets $\mathcal{J}^{\prime} \subseteq \mathcal{J}$ the projection $\prod_{A \in \mathcal{J}} M^{\preceq A} \rightarrow$ $\prod_{A \in \mathcal{J}^{\prime}} M^{\preceq A}$ along the decomposition obviously yields a homomorphism $M^{\mathcal{J}} \rightarrow$ $M^{\mathcal{J}^{\prime}}$ of $\overline{\mathcal{Z}}$-modules. We say that $M$ is a flabby $(\mathscr{A}, \preceq)$-cofiltered $\overline{\mathcal{Z}}$-module if, for any pair $\left(\mathcal{J}^{\prime}, \mathcal{J}\right)$ with $\mathcal{J}^{\prime} \subseteq \mathcal{J}$, the homomorphism $M^{\mathcal{J}} \rightarrow M^{\mathcal{J}^{\prime}}$ is surjective.

3.2. The support condition. We need one more condition on our objects. First, we define, for any $A \in \mathscr{A}$ and any cofiltered $\overline{\mathcal{Z}}$-module $M$, the $\overline{\mathcal{Z}}$-module

$$
M_{[A]}:=\operatorname{ker}\left(M^{\preceq A} \rightarrow M^{\prec A}\right) .
$$

Note that there is a unique $w \in \mathcal{W}$ with $w\left(A_{e}\right) \in A+\mathbb{Z} R$. We denote this $w$ by $\pi(A)$, and in this way we obtain a map $\pi: \mathscr{A} \rightarrow \mathcal{W}$. We say that $M$ satisfies the support condition if for any $A \in \mathscr{A}$, the action of $\left(z_{w}\right) \in \overline{\mathcal{Z}}$ on $M_{[A]}$ is given by multiplication with the scalar $z_{\pi(A)}$.

We denote by $\overline{\mathcal{Z}}$-mod ${ }^{\preceq}$ the category of all $(\mathscr{A}, \preceq)$-cofiltered $\overline{\mathcal{Z}}$-modules that satisfy the following assumptions:

(1) $M$ is flabby.

(2) $M$ satisfies the support condition.

(3) For all open subset $\mathcal{J}$, the $S$-module $M^{\mathcal{J}}$ is finitely generated and torsion free.

The following two subcategories are then important for us:

- $\mathcal{B}^{\text {ref }} \subset \overline{\mathcal{Z}}$-mod ${ }^{\preceq}$ is the full subcategory of objects that have the property that $M^{\mathcal{J}}$ is a reflexive $S$-module for any open subset $\mathcal{J}$. 
- $\mathcal{B} \subset \overline{\mathcal{Z}}$-mod $\preceq$ is the full subcategory of objects that have the property that $M^{\mathcal{J}}$ is graded free over $S$ for any open subset $\mathcal{J}$.

3.3. Projective objects and the Braden-MacPherson algorithm. Neither of the categories $\overline{\mathcal{Z}}$-mod ${ }^{\preceq}, \mathcal{B}^{\text {ref }}$ or $\mathcal{B}$ is abelian. But they do carry a natural exact structure instead.

Definition 3.1. We say that a sequence $0 \rightarrow M \rightarrow N \rightarrow O \rightarrow 0$ in $\overline{\mathcal{Z}}$-mod $\stackrel{\preceq}{\text { is }}$ exact, if for any open subset $\mathcal{J}$ of $\mathscr{A}$ the induced sequence

$$
0 \rightarrow M^{\mathcal{J}} \rightarrow N^{\mathcal{J}} \rightarrow O^{\mathcal{J}} \rightarrow 0
$$

is an exact sequence of $\overline{\mathcal{Z}}$-modules.

One checks that this indeed defines an exact structure in the sense of Quillen. This exact structure allows us to talk about projective objects in the categories $\overline{\mathcal{Z}}$-mod ${ }^{\preceq}, \mathcal{B}^{\text {ref }}$ and $\mathcal{B}$. Note that we call an object $P$ in either of these categories projective if the respective $\operatorname{Hom}(P, \cdot)$ functor maps a short exact sequence to a short exact sequence of abelian groups.

We have the following result:

Theorem 3.2 ([FL1]). For each $A \in \mathscr{A}$ there is an up to isomorphism unique object $B(A)$ in the category $\mathcal{B}^{\text {ref }}$ with the following properties:

(1) $B(A)$ is indecomposable and projective.

(2) $B(A)_{[B]}=0$ unless $A \preceq B$, and $B(A)_{[A]} \cong S$.

In the paper [FL1], the object $B(A)$ is obtained by taking global sections of an $(\mathscr{A}, \preceq)$-cofiltered sheaf on the finite moment graph $\mathcal{G}$ associated with the root system $R$. This sheaves is constructed algorithmically by a cofiltered version of the Braden-MacPherson algorithm (cf. [BMP01]). It is not at all clear from the construction that $B(A)$ admits a Verma flag. Still the objects $B(A)$ are characterized by the projectivity, and one can construct them locally, i.e. vertex by vertex, using a linear-algebraic algorithm.

3.4. Translation functors and a duality. In the paper [FL2] an alternative proof of the above statement is presented. In this paper we introduce and study translation functors on the categories $\overline{\mathcal{Z}}$-mod ${ }^{\preceq}, \mathcal{B}^{\text {ref }}$ and $\mathcal{B}$, and we obtain an additional property of the objects $B(A)$.

Theorem 3.3 ([FL2]). For each $A \in \mathscr{A}$, the object $B(A)$ admits a Verma flag, i.e. it is contained in $\mathcal{B}$.

Moreover, we introduce a duality functor D associated with the longest element $w_{0}$ in the finite Weyl group and we prove the following

Theorem 3.4 ([FL2]). For each alcove $A$ we have

$$
\mathrm{D} B(A) \cong B\left(w_{0}(A)\right)[\ell(A)],
$$

where $\ell: \mathscr{A} \rightarrow \mathbb{Z}$ is the length function introduced by Lusztig. 
3.5. Connection to the Andersen-Jantzen-Soergel category. In [FL3] we define a functor $\Psi: \overline{\mathcal{Z}}$-mod $\preceq \rightarrow \mathcal{K}$. The main steps in the construction are the following. Let $M$ be an object in $\overline{\mathcal{Z}}$-mod $\preceq$. For an alcove $A$ we set

$$
\Psi(M)(A):=\left(M_{[A]}\right)^{\emptyset} .
$$

For a positive root $\alpha$ we consider the $\preceq$-interval $[A, \alpha \uparrow A]$. Then there is a unique direct summand of $\left(M_{[A, \alpha \uparrow A]}\right)^{\emptyset}$ canonically isomorphic to $M_{[A]}^{\emptyset} \oplus M_{[\alpha \uparrow A]}^{\emptyset}$, and we denote by $p$ the projection. We then define

$$
\Psi(M)(A, \alpha):=\operatorname{im}\left(\left(M_{[A, \alpha \uparrow A]}\right)^{\alpha} \rightarrow\left(M_{[A, \alpha \uparrow A]}\right)^{\emptyset} \stackrel{p}{\rightarrow} M_{[A]}^{\emptyset} \oplus M_{[\alpha \uparrow A]}^{\emptyset}\right) .
$$

In [FL3] we then show:

Theorem 3.5. The image under the functor $\Psi$ of the subcategory $\mathcal{B}^{\text {proj }}$ of projective objects in $\mathcal{B}$ is the Andersen-Jantzen-Soergel subcategory $\mathcal{M}$ of $\mathcal{K}$.

The above theorem hence provides the more intrinsic definition of the category $\mathcal{M}$ that we were looking for.

3.6. Multiplicities and periodic polynomials. In [FL3] we introduce another functor. It takes a Braden-MacPherson sheaf on an affine moment graph and

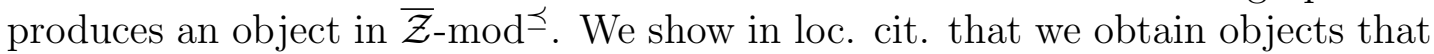
are projective in $\mathcal{B}$. Even though the functor is not fully faithful it yields enough structure for a proof of the following result.

Theorem 3.6. Let $A \in \mathscr{A}$ be an alcove, and assume that either $\operatorname{ch} k=0$ or $\operatorname{ch} k$ is big enough. Then

$$
\operatorname{rk}_{S} B(A)_{[C]}=p_{C, A}(1)
$$

for any $C \in \mathscr{A}$.

By "big enough" we mean that there exists a number $N$ such that the statement of the theorem is true if $\operatorname{ch} k>N$. In fact, the theorem above holds if the corresponding affine Kazhdan-Lusztig conjecture holds for the Braden-MacPherson sheaf $\mathscr{B}(w)$ on the affine moment graph over $k$, for all $w$ such that $w\left(A_{e}\right)$ is contained in the antifundamental box, i.e the set of vectors $\lambda \in V$ such that $-1 \leqslant\left\langle\lambda, \alpha^{\vee}\right\rangle \leqslant 0$ for any $\alpha \in \Pi$.

Now if $p$ is big enough in the sense of Theorem 3.6, then we obtain from the above, the definition of $\Psi$ and the Andersen-Jantzen-Soergel result in Theorem 2.3 that

$$
[Z(w .0): L(x .0)]=\operatorname{rk}_{S^{\emptyset}} Q_{A_{w_{0} x}}\left(A_{w_{0} w}\right) \leqslant \operatorname{rk}_{S} B\left(A_{w_{0} x}\right)_{\left[A_{w_{0}}\right]}=p_{A_{w_{0} w}, A_{w_{0} x}}(1)
$$

for all $w, x \in \widehat{\mathcal{W}}$. Once this is established, it is easy to obtain the reverse inequality, and hence

$$
[Z(w .0): L(x .0)]=p_{A_{w_{0} w}, A_{w_{0} x}}(1)
$$

which is by what we explained in Section 2.7 equivalent to Lusztig's conjecture. 


\section{REFERENCES}

[AJS94] Henning Haahr Andersen, Jens Carsten Jantzen, and Wolfgang Soergel, Representations of quantum groups at a pth root of unity and of semisimple groups in characteristic p: independence of p, Astérisque (1994), no. 220, 321.

[BMP01] T. Braden, R. MacPherson, From moment graphs to intersection cohomology, Math. Ann. 321 (2001), no. 3, 533-551.

[FL1] Peter Fiebig and Martina Lanini, Filtered moment graph sheaves, preprint 2015, arXiv: 1508.05579.

[FL2] Peter Fiebig and Martina Lanini, Periodic structures on affine moment graphs I: Dualities and translation functors, preprint 2015, arXiv:1504.01699.

[FL3] Peter Fiebig and Martina Lanini, Periodic structures on affine moment graphs II: Multiplicities and modular representations, in preparation.

[Fie08] Peter Fiebig, Sheaves on moment graphs and a localization of Verma flags, Adv. Math. 217 (2008), 683-712.

[Fie10] _ Lusztig's conjecture as a moment graph problem, Bull. London Math. Soc. 42(6) (2010), 957-972.

[Jan77] Jens Carsten Jantzen, Über das Dekompositionsverhalten gewisser modularer Darstellungen halbeinfacher Gruppen und ihrere LIe Algebren, J. Algebra 49

[Lus80a] George Lusztig, Some problems in the representation theory of finite Chevalley groups, The Santa Cruz Conference on Finite Groups (Univ. California, Santa Cruz, Calif., 1979), Proc. Sympos. Pure Math., vol. 37, Amer. Math. Soc., Providence, R.I., 1980, pp. 313-317.

[Lus80b] _ Hecke algebras and Jantzen's generic decomposition patterns, Adv. Math. 37 (1980), no. 2, 121-164.

[Soe97] Wolfgang Soergel, Kazhdan-Lusztig-Polynome und eine Kombinatorik für KippModuln, Represent. Theory 1 (1997), 37-68 (electronic).

[Wil] Geordie Williamson, Schubert calculus and torsion explosion, preprint 2013, arXiv:1309.5055.

Department Mathematik, FAU Erlangen-NÜrnberg, Cauerstrasse 11, 91058 ERLangen, Germany

E-mail address: fiebig@math.fau.de

School of Mathematics, University of Edinburgh, Edinburgh EH9 3FD, UK

E-mail address: m.lanini@ed.ac.uk 